\title{
Comparison of Epiretinal Membrane Peel after Dual Staining Versus En Bloc Peel Using Negative Staining Technique
}

\author{
Tehmina Jahangir ${ }^{1}$, Qasim Lateef Chaudhry ${ }^{2}$, Haroon Tayyab ${ }^{3}$ \\ ${ }^{1-2}$ Department of Ophthalmology, Allama lqbal Medical College, Lahore, ${ }^{3}$ Department of Ophthalmology, Mayo \\ Hospital, KEMU Lahore
}

\begin{abstract}
Purpose: To compare the completeness of ILM peel in cases of Idiopathic Epiretinal Membranes following double staining and double peeling versus en bloc dissection of both using negative staining method.

Study Design: Quasi experimental study.

Place and Duration of Study: Jinnah Hospital Lahore, from October 2017 to March 2018.

Material and Methods: Forty eyes of forty patients with Idiopathic Epiretinal membranes (ERM) were selected by non-probability convenient method and divided into two groups. In group A, the ERM was initially stained with Trypan blue and then peeled. Subsequently the macula was re-stained with Brilliant blue G (BBG). In group B, ERM and ILM were successfully peeled together as a single membrane utilizing the single block technique, after a desired negative staining effect was obtained by injecting BBG. The thoroughness of the ILM peel was established using another injection of BBG although a few remains were often identified outside the central macular area, no other dyes were used.
\end{abstract}

Results: In group A, 8 of the 20 eyes (40\%) had full undisturbed ILM present whereas in 10 eyes (50\%) the ILM was there but damaged, only 2 eyes (10\%) had near total ILM removed. In group B, the dual peeling was successful in 17 of the 20 cases (85\%). There has been no recurrence of ERM until now.

Conclusion: Instead of peeling twice, the single en bloc negative staining technique with Brilliant blue $\mathrm{G}$ is a safer and more effective method for removal of ERM together with ILM.

Key Words: Pars plana vitrectomy, Epiretinal membrane, Internal limiting membrane.

How to Cite this Article: Jahangir T, Chaudhry QL, Tayyab H. Comparison of Residual Internal Limiting Membrane Following Epiretinal Membrane Peel After Dual Staining Versus En Bloc Peel Using Negative Staining Technique, Pak J Ophthalmol. 2020; 36 (1): 19-23.

DOI: https://doi.org/10.36351/pjo.v36i1.897

\section{INTRODUCTION}

Epiretinal membrane (ERM) is a disorder of the vitreomacular interface and is used to describe a condition in which there is cellular proliferation on the inner retinal surface. Epiretinal membrane is a very

Address for Correspondence: Tehmina Jahangir Assistant Professor Ophthalmology Department Allama Iqbal Medical College, Lahore E-mail: tehminajahangir@gmail.com common finding in people over the age of 50 years. The prevalence of Idiopathic ERM is $2 \%$ in people younger than 60 years and approximately $12 \%$ in those who are more than 70 years of age ${ }^{1}$. Most are discovered incidentally during routine ophthalmoscopic examination. Others become symptomatic and require treatment. The standard of treatment for a symptomatic ERM is its surgical removal. Modern vitreoretinal techniques involve small-gauge vitrectomy and the use of dyes to 
facilitate the visualization of membrane ${ }^{1}$. Several studies have advocated peeling of ILM during ERM surgery to minimize the recurrence of ERM without negatively affecting visual outcomes ${ }^{2,3}$. It has been suggested that ILM removal per-operatively removes the scaffolding for any fibroblast proliferation and residual microscopic ERM, therefore diminishing the risk of recurrence in addition to improving the final visual outcomes ${ }^{1}$.

We conducted a prospective interventional study to compare the completeness of ILM peel using two different methods of ERM staining.

\section{MATERIAL AND METHODS}

We studied 40 consecutive patients (40 eyes), 22 males and 28 females who underwent surgery for primary idiopathic epiretinal membrane over a period of 6 months from October 2017 to March 2018. All patients were followed up for $\geq 6$ months after they were enrolled in the study. All ophthalmic surgeries were performed by one ophthalmic surgeon (XY) at the Department of Ophthalmology, Jinnah Hospital, Lahore. Patients with secondary ERM, presence of glaucoma, retinal degeneration, optic neuropathy and age less than 18 years were excluded from the study. Approval for the study was obtained by the hospital ethics committeeand informed consent was taken in writing from all subjects.

A standard subtotal 23G 3-ports pars plana vitrectomy (PPV) was performed using the Optikon Pulsar 2 Vitrectomy system. The microscope in use was Leica M844 F20 with its built-in RUV 800 viewing system.

In Group A, a standard 23G PPV was performed. After performing core vitrectomy and inducing posterior vitreous detachment, fluid air exchange was done and ERM was stained with Trypan blue dye. The fluid was reopened through the infusion cannula and the stained ERM was peeled with $23 \mathrm{G}$ intraocular endgripping forceps. BBG $(0.025 \%)$ was applied to the peeled area (under air) for another one minute and the extent of the residual ILM was noted. A second peeling of the ILM was performed using ILM forceps in all cases with residual ILM whether it was intact or damaged.

In Group B, a standard 23G PPV was performed. After core vitrectomy and induction of PVD, fluid air exchange was done. We proceeded with a singular technique of staining by introducing the BBG dye
$0.025 \%$, through either of the superior ports. The injected dye was directed towards the macula but injected in a very gentle manner while keeping a safe distance. The dye was left in place under air for a minute before reopening the fluid. Since BBG primarily stains the ILM it did not stain the areas concealed by the ERM. Therefore, in this group we aimed to stain the ERM negatively while staining the ILM directly, so the contrasting margins between the unstained ERM and the stained ILM were clearly distinguished thus achieving a negative staining effect (fig. 1). With a $23 \mathrm{G}$ ILM peeling forceps the ILM was pinched and peeled temporally simultaneously double peeling i.e., dragging enbloc the ERM and the underlying ILM and in a single step in an annular direction around the fovea (fig. 2). In almost all cases, the peel was extended up to the temporal vascular arcades releasing any centripetal and tangential tractions along the way. We then reinjected the BBG to re-stain the peeled area to confirm any residual ILM requiring removal (fig. 3 and 4). All cases in both groups were closed under SF6 tamponade.

All data was recorded on a predesigned proforma. The data was entered and analyzed by SPSS (version 20) using the Chi Square test for statistical analysis and results.

\section{RESULTS}

In group A, the ERM was initially stained with Trypan blue, the ERM was peeled. Subsequently the macula was re-stained with BBG. Eight of the 20 eyes (40\%) had full undisturbed ILM whereas in 10 eyes $(50 \%)$ the ILM was there but damaged, only 2 eyes (10\%) had near total ILM removed.

In group $\mathrm{B}$, the dual peeling was successfully performed in 17 of the 20 cases $(85 \%)$ by utilizing the en block technique, after successfully obtaining a negative staining effect using BBG. The completeness of ILM peel was established using an additional BBG injection although some residual ILM was often encountered further away from the central macular area. No other dye was injected. There has been no recurrence of ERM to date.

In either of the two groups there were no significant complications related to peeling except for a few superficial self-resolving retinal hemorrhages and one case of small iatrogenic hole in the temporal macula. 


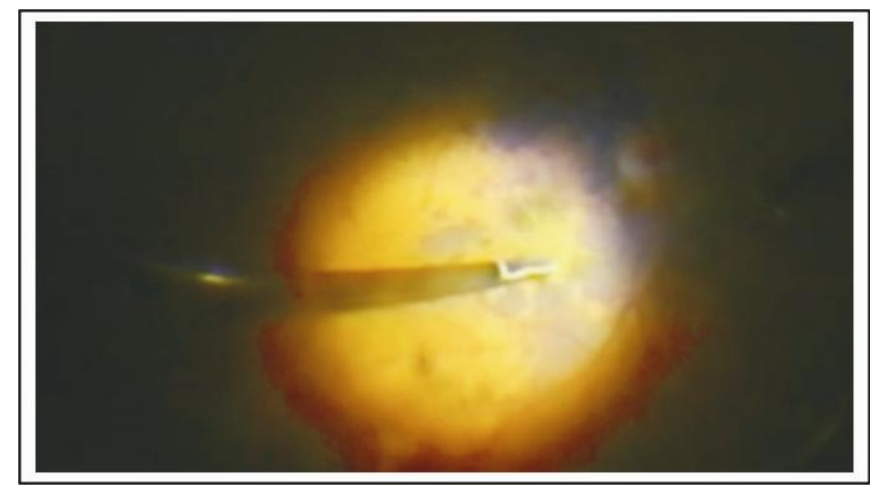

Fig. 1: Negative staining effect: the blue areas represent the internal limiting membrane as stained with the Brilliant Blue $G$ dye; Areas devoid of the dye are covered with the epiretinal membrane.

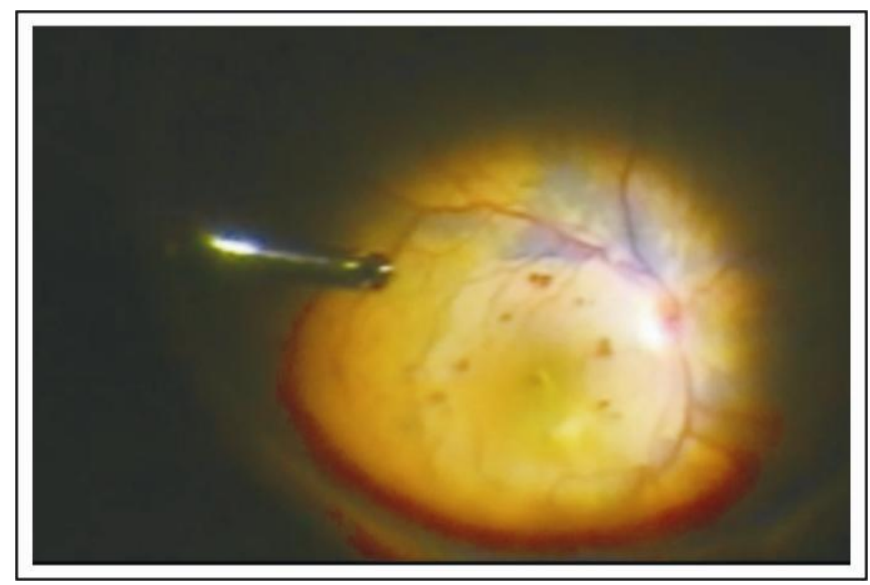

Fig. 2: Enbloc peeling: both the internal limiting membrane and the epiretinal membrane are removed in a single step.

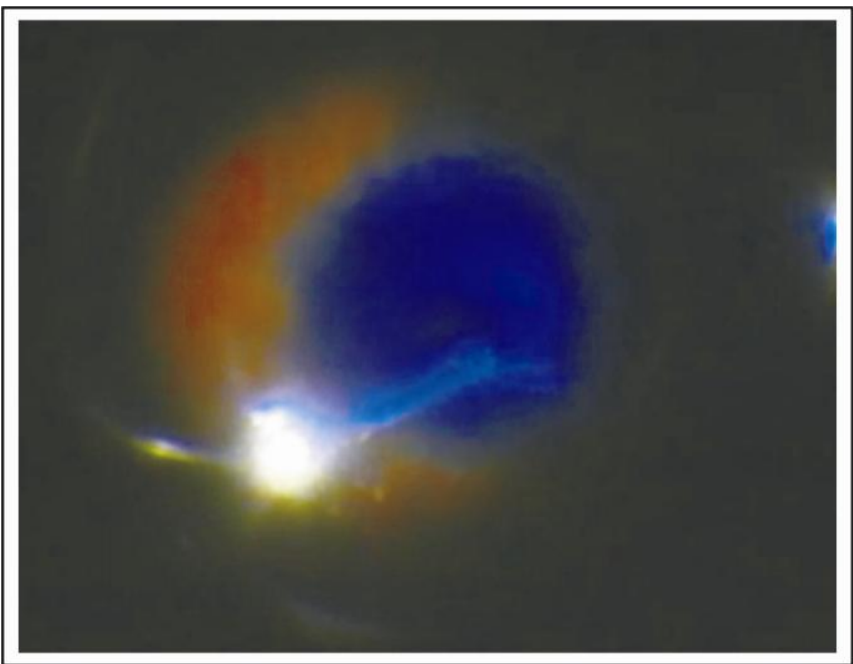

Fig. 3: Second time Brilliant Blue G dye injected near the macula.

All data was recorded on a predesigned proforma. The data was entered and analyzed by SPSS (version 20).

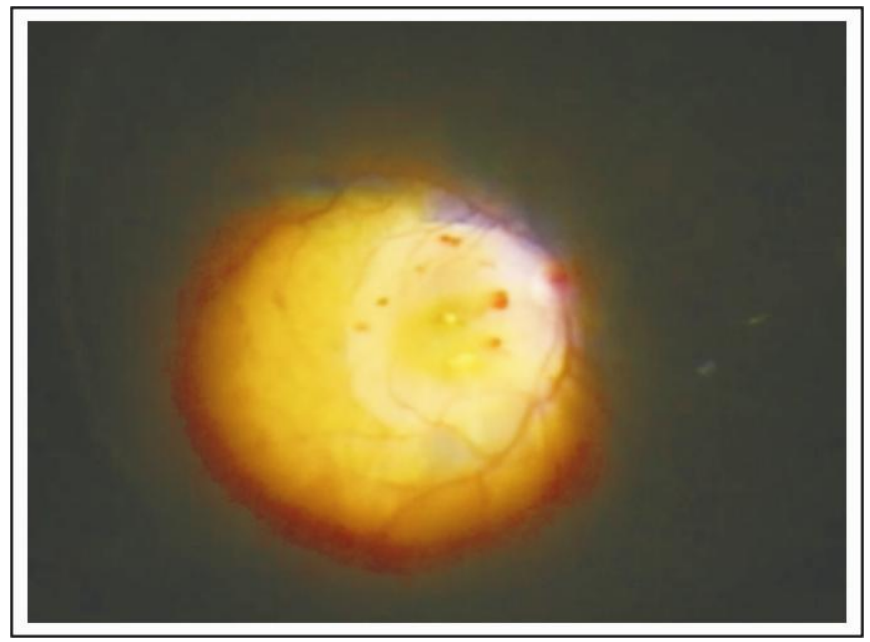

Fig. 4: No residual Internal limiting membrane can be seen after en bloc removal of both membranes in a single step.

\section{DISCUSSION}

ERM usually requires removal when it leads to structural damage to the retina, an increase in macular thickness or troublesome visual symptoms. Ideally the surgery for ERM removal should both have a minimal recurrence and little or no retinal trauma and toxicity ${ }^{4,5}$. This can be accomplished by simultaneously peeling the ILM and by using the least toxic dye in as little a concentration as feasible.

The rate of ERM recurrence is lower when combined with ILM peel ${ }^{6}$. The postoperative BCVA or CMT is not influenced significantly by ILM peeling which endorses the use of the surgical technique for patients with $\mathrm{ERM}^{7,8}$. Moreover, ILM peeling has a clear advantage of decreasing ERM recurrence ${ }^{9,10}$.

Although in 30 to $70 \%$ of patients, ILM is often removed together with ERM, complete and sufficient area of ILM peeling is essential to avoid ERM recurrence $^{11}$.

The technique of negative staining reported by Foster et al ${ }^{12}$ aimed to decrease injury to the retina and increase visibility, by utilizing indocyanine green (ICG) to preferentially stain the ILM surrounding the margins of the ERM, although it lightly stained the ERM as well. However, when selecting dyes that preferentially stain the ILM, recent studies suggest that BBG may be the least toxic, especially in comparison to ICG $^{13,14}$. Brilliant Blue $G$ is a selective dye used to stain the ILM on a stand-alone basis, with little to no affinity for the ERM, and a suitable safety profile ${ }^{15}$. Moreover, current evidence suggests that this dye may also have a protective role against cell apoptosis ${ }^{16}$. The 
results of our study are comparable with those of Carpentier et al and Shimada ${ }^{2,3}$.

Removal of the two membranes in a single step has several advantages. It reduces the number of times the retina is grasped as well as traction on the retinal surface while simultaneously reducing the membrane removal time and diminishing phototoxicity; all of which are probable causes of iatrogenic damage to the retina $^{17,18}$.

Although the availability of stains for the ILM has made peeling safer, the procedure is still not free of complications. These include: retinal edema, nerve fiber layer damage, retinal hemorrhages, electrophysiological shifts and visual field changes have all been documented after ILM peeling ${ }^{19,20,21}$.

The limitation of our study is the small sample size. Research with large sample size and data from different hospitals will be helpful in further proving the results.

\section{CONCLUSION}

Instead of using two dyes for staining and peeling of ERM twice, using a single en bloc technique is not only less time-consuming but also safer in terms of decreased exposure to dyes, reduction in grasping attempts and overall reduced surgical time as well as decreased phototoxicity.

\section{Ethical Approval}

The study was approved by the Institutional review board/Ethical review board.

\section{Conflict of Interest}

Authors declared no conflict of interest

\section{Authors' Designation and Contribution}

Tehmina Jahangir; Assistant Professor: Study design, manuscript writing, literature review.

Qasim Latif Chaudhary; Associate Professor: Literature review and final critical review.

Haroon Tayyab; Assistant Professor: Literature review and final critical review.

\section{REFERENCES}

1. Wickham L, Konstantinidis L, Thomas J. Wolfensberge. Macular Epiretinal Membranes. In:
Ryan SJ, Wilkinson CP, editors. Retina - Vol III, Surgical Retina. Philadelphia, PA: Elsevier Inc.; 2018. p. 2261-2279.

2. Carpentier C, Zanolli M, Wu L, Sepulveda G, Berrocal MH, Saravia M, et al. Pan-American Collaborative Retina Study Group. Residual internal limiting membrane after epiretinal membrane peeling: results of the Pan-American Collaborative Retina Study Group. Retina. 2013; 33 (10): 2026-2031.

3. Shimada $\mathbf{H}$, Nakashizuka $\mathbf{H}$, Hattori $\mathbf{T}$, Mori $\mathbf{R}$, Mizutani Y, Yuzawa M. Double staining with brilliant blue $\mathrm{G}$ and double peeling for epiretinal membranes. Ophthalmology, 2009; 116 (7): 1370-1376.

4. Liu H., Zuo S., Ding C., Dai X., Zhu X. Comparison of the effectiveness of pars plana vitrectomy with and without internal limiting membrane peeling for idiopathic retinal membrane removal: a meta-analysis. J Ophthalmol. 2015; 2015: 974568.

Doi:10.1155/2015/974568.

5. Gandorfer A, Haritoglou C, Scheler R, Schumann R, Zhao F, Kampik A. Residual cellular proliferation on the internal limiting membrane in macular pucker surgery. Retina. 2012; 32 (3): 477-485.

6. Bovey EH, Uffer S, Achache F. Surgery for epimacular membrane: impact of retinal internal limiting membrane removal on functional outcome. Retina, 2004; 24 (5): 728-735.

7. Geerts L, Pertile G, van de Sompel W, Moreels T, Claes C. Vitrectomy for epiretinal membranes: visual outcome and prognostic criteria. Bull Soc Belge Ophtalmol. 2004; 293: 7-15.

8. Jung JJ, Hoang QV, Ridley-Lane ML, Sebrow DB, Dhrami-Gavazi E, Chang S. Long-term retrospective analysis of visual acuity and optical coherence topographic changes after single versus double peeling during vitrectomy for macular epiretinal membranes. Retina, 2016; 36 (11): 2101-2109.

9. Sandali O, El Sanharawi M, Basli E, Bonnet S, Lecuen N, Barale PO, et al. Epiretinal membrane recurrence: incidence, characteristics, evolution, and preventive and risk factors. Retina, 2013; 33 (10): 2032-2038.

10. Oh HN, Lee JE, Kim HW, Yun IH. Clinical outcomes of double staining and additional ILM peeling during ERM surgery. Korean J Ophthalmol. 2013; 27 (4): 256-260.

11. Chang S, Gregory-Roberts EM, Park S, Laud K, Smith SD, Hoang QV. Double peeling during vitrectomy for macular pucker: the Charles L. Schepens Lecture. JAMA Ophthalmol. 2013; 131 (4): 525-530.

12. Foster RE, Petersen MR, Da Mata AP, Burk SE, Rosa RH Jr, Riemann CD. Negative indocyanine green staining of epiretinal membranes. Retina, 2002; 22 (1): 106-108.

13. Enaida H, Hisatomi T, Hata Y, Ueno A, Goto Y, 
Yamada T, et al. Brilliant blue G selectively stains the internal limiting membrane/brilliant blue Gassisted membrane peeling. Retina, 2006; 26 (6): 631-6.

14. Kawahara S, Hata Y, Miura M, Kita T, Sengoku A, Nakao S, et al. Intracellular events in retinal glial cells exposed to ICG and BBG. Invest Ophthalmol Vis Sci. 2007; 48: 4426-32.

15. Azuma K, Noda $\mathbf{Y}$, Hirasawa $\mathbf{K}$, Ueta T. Brilliant blue $\mathrm{G}$ assisted internal limiting membrane peeling for macular hole: a Systematic Review of Literature and Meta-Analysis. Retina, 2016; 36 (5): 851-858.

16. Remy M, Thaler S, Schumann RG, May CA, Fiedorowicz M, Schuettauf F, et al. An in vivo evaluation of brilliant blue $\mathrm{G}$ in animals and humans. Br J Ophthalmol. 2008; 92 (8): 1142-7.

17. Azuma K, Ueta T, Eguchi S, Aihara M. Effects of Internal Limiting Membrane Peeling Combined with
Removal of Idiopathic Epiretinal Membrane: A Systemic Review of Literature and Meta-Analysis. Retina, 2017; 37 (10): 1-7.

18. Pournaras CJ, Emarah A, Petropoulos IK. Idiopathic macular epiretinal membrane surgery and ILM peeling: anatomical and functional outcomes. Semin Ophthalmol. 2011; 26 (2): 42-46.

19. Liu Y, Kato C, Herai T. Evaluation of peeling of inner limiting membrane for idiopathic epiretinal membrane. Jpn J Clin Ophthalmol. 2001; 55: 1239-1243.

20. Wu L. Epiretinal Membrane Surgery: To peel or not to peel the ILM? Retina Today. 2017: 30-32.

21. Roh M, Eliott D. Internal limiting membrane peeling during idiopathic epiretinal membrane removal: literature review. Int Ophthalmol Clin. 2015; 55 (4): 91-101.

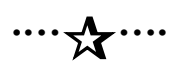

